\title{
Comment on the paper by A. H. Joarder
}

\section{Saralees Nadarajah}

Published online: 19 December 2007

(C) Springer-Verlag 2007

The recent paper by Joarder (2007) studied the moments of the product and ratio of two correlated chi-square variables. The main results of the paper are presented in Theorems 2.1, 3.1 and 4.1. Theorem 2.1 derives the exact probability density function (pdf) of the two correlated chi-square variables in the form:

$$
f\left(u_{1}, u_{2}\right)=A\left(u_{1}, u_{2}\right) \sum_{k=0}^{\infty}\left\{1+(-1)^{k}\right\}\left(\frac{\rho \sqrt{u_{1} u_{2}}}{1-\rho^{2}}\right)^{k} \frac{\Gamma((k+1) / 2)}{k ! \Gamma((k+m) / 2)},
$$

where

$$
A\left(u_{1}, u_{2}\right)=\frac{2^{-m-1}\left(u_{1} u_{2}\right)^{m / 2-1} \exp \left\{-\left(u_{1}+u_{2}\right) /\left(2\left(1-\rho^{2}\right)\right)\right\}}{\sqrt{\pi} \Gamma(m / 2)\left(1-\rho^{2}\right)^{m / 2}} .
$$

Theorem 3.1 derives the product moment of the two correlated chi-square variables in the form:

$$
\begin{aligned}
E\left(U_{1}^{a} U_{2}^{b}\right)= & B \sum_{k=0}^{\infty}\left\{1+(-1)^{k}\right\} \frac{(2 \rho)^{k}}{k ! \Gamma((k+m) / 2)} \Gamma\left(\frac{k+m}{2}+a\right) \\
& \times \Gamma\left(\frac{k+m}{2}+b\right) \Gamma\left(\frac{k+1}{2}\right),
\end{aligned}
$$

The online version of the original article can be found at doi:10.1007/s00362-007-0105-0

S. Nadarajah $(\varangle)$

School of Mathematics, University of Manchester, Manchester M601QD, UK

e-mail: saralees.nadarajah@manchester.ac.uk 
where

$$
B=\frac{2^{a+b-1}\left(1-\rho^{2}\right)^{a+b+m / 2}}{\sqrt{\pi} \Gamma(m / 2)} .
$$

Theorem 4.1 derives the pdf of the correlation coefficient for a bivariate $t$ population in the form:

$$
h(r)=C(r) \sum_{k=0}^{\infty} \frac{(2 \rho r)^{k}}{k !} \Gamma^{2}\left(\frac{k+m}{2}\right),
$$

where

$$
C(r)=\frac{2^{m-2}\left(1-\rho^{2}\right)^{m / 2}\left(1-r^{2}\right)^{(m-3) / 2}}{\pi \Gamma(m-1)} .
$$

Each of (1), (2) and (3) involves an infinite sum. It appears that Joarder (2007) made no attempts to simplify the infinite sums. Here, I would like to point out that each of (1), (2) and (3) can be reduced to known hypergeometric functions. This can be handy since in-built routines for the hypergeometric functions are widely available.

First consider (1). Note that

$$
\begin{aligned}
f\left(u_{1}, u_{2}\right) & =2 A\left(u_{1}, u_{2}\right) \sum_{k=0}^{\infty}\left(\frac{\rho \sqrt{u_{1} u_{2}}}{1-\rho^{2}}\right)^{2 k} \frac{\Gamma(k+1 / 2)}{(2 k) ! \Gamma(k+m / 2)} \\
& =2 \sqrt{\pi} A\left(u_{1}, u_{2}\right) \sum_{k=0}^{\infty}\left(\frac{\rho^{2} u_{1} u_{2}}{4\left(1-\rho^{2}\right)^{2}}\right)^{k} \frac{1}{k ! \Gamma(k+m / 2)} \\
& =\frac{2 \sqrt{\pi} A\left(u_{1}, u_{2}\right)}{\Gamma(m / 2)}{ }_{0} F_{1}\left(; \frac{m}{2} ; \frac{\rho^{2} u_{1} u_{2}}{4\left(1-\rho^{2}\right)^{2}}\right)
\end{aligned}
$$

where ${ }_{0} F_{1}(; a ; x)$ is the hypergeometric function defined by

$$
{ }_{0} F_{1}(; a ; x)=\sum_{n=0}^{\infty} \frac{1}{(a)_{n}} \frac{x^{n}}{n !},
$$

where $(f)_{n}=\Gamma(f+n) / \Gamma(f)$ denotes the ascending factorial. Now consider (2). Note that

$$
\begin{aligned}
E\left(U_{1}^{a} U_{2}^{b}\right) & =2 B \sum_{k=0}^{\infty} \frac{(2 \rho)^{2 k}}{(2 k) ! \Gamma(k+m / 2)} \Gamma\left(k+\frac{m}{2}+a\right) \Gamma\left(k+\frac{m}{2}+b\right) \Gamma\left(k+\frac{1}{2}\right) \\
& =2 \sqrt{\pi} B \sum_{k=0}^{\infty} \frac{\rho^{2 k}}{k ! \Gamma(k+m / 2)} \Gamma\left(k+\frac{m}{2}+a\right) \Gamma\left(k+\frac{m}{2}+b\right) \\
& =\frac{2 \sqrt{\pi} B \Gamma(m / 2+a) \Gamma(m / 2+b)}{\Gamma(m / 2)}{ }_{2} F_{1}\left(\frac{m}{2}+a, \frac{m}{2}+b ; \frac{m}{2} ; \rho^{2}\right)
\end{aligned}
$$


where ${ }_{2} F_{1}(a, b ; c ; x)$ is the Gauss hypergeometric function defined by

$$
{ }_{2} F_{1}(a, b ; c ; x)=\sum_{n=0}^{\infty} \frac{(a)_{n}(b)_{n}}{(c)_{n}} \frac{x^{n}}{n !}
$$

Finally, consider (3). Note that

$$
\begin{aligned}
h(r)= & C(r)\left[\sum_{k=0}^{\infty} \frac{(2 \rho r)^{2 k}}{(2 k) !} \Gamma^{2}\left(k+\frac{m}{2}\right)+\sum_{k=0}^{\infty} \frac{(2 \rho r)^{2 k+1}}{(2 k+1) !} \Gamma^{2}\left(k+\frac{m+1}{2}\right)\right] \\
= & \sqrt{\pi} C(r)\left[\sum_{k=0}^{\infty} \frac{\left(\rho^{2} r^{2}\right)^{k}}{k !} \frac{\Gamma^{2}(k+m / 2)}{\Gamma(k+1 / 2)}+\rho r \sum_{k=0}^{\infty} \frac{\left(\rho^{2} r^{2}\right)^{k}}{k !} \frac{\Gamma^{2}(k+(m+1) / 2)}{\Gamma(k+3 / 2)}\right] \\
= & C(r)\left[\Gamma^{2}\left(\frac{m}{2}\right){ }_{2} F_{1}\left(\frac{m}{2}, \frac{m}{2} ; \frac{1}{2} ; \rho^{2} r^{2}\right)\right. \\
& \left.+2 \rho r \Gamma^{2}\left(\frac{m+1}{2}\right){ }_{2} F_{1}\left(\frac{m+1}{2}, \frac{m+1}{2} ; \frac{3}{2} ; \rho^{2} r^{2}\right)\right] .
\end{aligned}
$$

The hypergeometric functions in (4), (5) and (6) are well known and well established, see Prudnikov et al. (1986) for detailed properties. The nine corollaries-Corollaries 3.1 to 3.9 - of Joarder (2007) follow from (5) by using known properties of the Gauss hypergeometric function, see Sect. 7.3 of Prudnikov et al. (1986, vol 3).

\section{References}

Joarder AH (2007) Moments of the product and ratio of two correlated chi-square variables. Stat Pap (in press)

Prudnikov AP, Brychkov YA, Marichev OI (1986) Integrals and series, vols 1, 2 and 3. Gordon and Breach Science Publishers, Amsterdam 